\title{
El issue de la inmigración en los votantes de VOX en las Elecciones Generales de noviembre de 2019
}

\author{
Paloma Castro Martínez \\ UNIVERSIDADE DE SANTIAGO DE COMPOSTELA, GALICIA, ESPAÑA \\ paloma.castro2@usc.es \\ Diego Mo Groba \\ UNIVERSIDADE DE SANTIAGO DE COMPOSTELA, GALICIA, ESPAÑA \\ diego.mo.groba@usc.es
}

\begin{abstract}
Resumen: Si hay un tema que se ha asociado al auge de la extrema derecha en Europa occidental ha sido el issue de la inmigración. Mientras la mayoría de los autores han observado la presencia de actitudes hacia la inmigración entre los votantes de estos partidos políticos distintas de las del resto de votantes, algunos autores han llegado a denominar a estas formaciones políticas como "partidos antinmigrantes" o "partidos racistas" (Boomgaarden y Vliegenthart, 2007; Fennema, 1996, 1997; Van der Brug y Fennema, 2003, 2005; Van Spanje, 2017). La inmigración ha sido el issue vertebrador del discurso político de la extrema derecha, en el que se entremezclan otros problemas como la cobertura de las prestaciones sociales y la viabilidad del Estado del bienestar, la economía y el desempleo, y la delincuencia y la seguridad. En este trabajo, tras exponer una profunda revisión bibliográfica sobre la relación entre la extrema derecha y la inmigración, se muestran las percepciones acerca de este issue de los votantes de VOX en las pasadas Elecciones Generales de noviembre de 2019, comicios en los que la extrema derecha se consolida en España al convertirse en la tercera fuerza política. En nuestro análisis exploratorio, se observa que los votantes de VOX muestran actitudes más hostiles hacia los inmigrantes y los issues relacionados con la inmigración que los votantes de resto de formaciones políticas.
\end{abstract}

Palabras clave: Extrema derecha, inmigración, single-issue, voto

Abstract: If there is one issue that has been associated with the rise of the extreme right in Western Europe, it has been the issue of immigration. While most authors have observed the presence of attitudes towards immigration among the voters of these political parties different from those of other voters, some authors have called these political formations as "anti-immigrant parties" or "racism parties" (Boomgaarden and Vliegenthart, 2007; Fennema, 1996, 1997; Van der Brug and Fennema, 2003, 2005; Van Spanje, 2017). Immigration has been the backbone of the political discourse of the extreme right, in which other problems such as the coverage of social benefits and the viability of the welfare state, the economy and unemployment, and crime and security are intertwined. In this paper, after exposing a deep bibliographic review on the relationship between the extreme right and immigration, we show the perceptions about this issue of VOX voters in the last General Elections of November 2019, elections in which the extreme right consolidates in Spain by becoming the third political force. In our exploratory analysis, it is observed that VOX voters show more hostile attitudes towards immigrants and immigration issues than voters from other political groups.

Keywords: extreme right, immigration, single-issue, vote 


\section{Introducción}

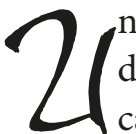

no de los issues más estudiados para explicar el renacer del voto a la extrema derecha en Europa occidental es la inmigración. No se trata sólo de las políticas antinmigración, sino de que este tema en sí mismo es el issue vertebrador del discurso político de la extrema derecha, en el que se entremezclan otros problemas como la cobertura de las prestaciones sociales y la viabilidad del Estado del bienestar, la economía y el desempleo, y la delincuencia y la seguridad. La inmigración funciona como un issue ómnibus, es decir, como un embudo a través del cual pasan muchas políticas, como la educación, la sanidad, la vivienda, el gasto público, los asuntos sociales o la cultura y los estilos de vida (Hainsworth, 2008: 70).

En nuestros días, la inmigración vuelve a ser un tema candente como consecuencia de la llamada "crisis de refugiados" de 2015 o del reciente movimiento "Black Lives Matter". Todas las democracias occidentales, además de estar afectadas, de diferentes formas, por estos sucesos, ven como las ideas defendidas por la extrema derecha se debaten en los círculos políticos y como sus políticas son adoptadas, de una forma más moderada, por los partidos del establishment, agitando así el estatus quo político nacional e internacional y aupando una ola de racismo e islamofobia sin precedentes. Y España ha dejado de ser la excepción europea.

Las elecciones autonómicas de 2018 suponen un punto de inflexión en el modelo político español. Por primera vez, en la historia de la democracia española, se produce la irrupción de un partido de extrema derecha: VOX, en sede parlamentaria, aceptándolo como parte negociadora en la conformación del gobierno andaluz. La presencia en el Parlamento andaluz catapulta a VOX al escenario nacional. Si tras las Elecciones Generales de abril de 2019, VOX obtiene 24 escaños en el Congreso de los diputados, tras la repetición de elecciones, se convierte en la tercera fuerza política con 52 escaños, sólo por detrás del Partido Socialista Obrero Español (PSOE) y del Partido Popular (PP).

El éxito cosechado por VOX abre una nueva área de investigación en la Ciencia Política española a fin de esclarecer qué factores han conducido al auge de la extrema derecha en nuestro país, así como cuáles son las características y las percepciones de los votantes de esta nueva organización política. En este marco, el presente trabajo trata de mostrar, de manera descriptiva, las percepciones que los votantes de VOX, en las Elecciones Generales de noviembre de 2019, tienen acerca de las cuestiones relacionadas con la inmigración, issue omnipresente en la mayoría de las investigaciones sobre la extrema derecha en Europa occidental. 


\section{La tesis del Single-issue}

El enfoque más desarrollado sobre el aumento del voto a los partidos de extrema derecha en Europa occidental es la tesis del single-issue (Arter, 1992). Los niche parties (partidos nicho), como Meguid (2005) los denomina, se diferencian de los partidos mainstreams en que politizan un conjunto de issues que, previamente, se encuentran fuera de las dimensiones de la competición partidista, retando, así, el contenido del debate político.

No obstante, los partidos de tema único no plantean temas nuevos. Aspecto crucial, pues los esfuerzos de la extrema derecha por acaparar y delimitar un issue pueden no conducir a los resultados esperados debido a que estos habían sido expuestos, con anterioridad, por los partidos tradicionales, de tal forma que los ciudadanos pueden tener ya definida su postura con respecto a ese tema. (Mudde, 2017). La extrema derecha no busca introducir nuevos issues, sino capitanear y reformular aquellos ya existentes en la arena política a fin de cristalizar al electorado potencial (Kitschelt, 1995).

La extrema derecha coopta un tema al que la mayoría de los políticos del establishment, tanto de derechas como de izquierdas, no han prestado la suficiente atención o con el que se muestran en desacuerdo (Zúquete, 2017), obligándolos a posicionarse al respecto. Así, si un partido de extrema derecha logra monopolizar un issue, los ciudadanos pueden percibir que esta formación política es la más efectiva para gestionarlo (Boomgaarden y Vliegenthart, 2007: 407).

Por su parte, los votantes son incapaces de recordar las propuestas políticas específicas, pero tienen una idea general de la orientación de las políticas que los partidos defienden. Los issues impactan en los apoyos electorales porque actúan como importantes indicadores emocionales (Maddens y Hajnal, 2001: 320). Particularmente, aquellos problemas no solucionados que afectan a la ciudadanía de forma directa causan emociones negativas: los problemas no sólo ocasionan frustración a las personas afectadas por estos, sino que también producen preocupación y ansiedad a los no afectados. Ante esta situación, los partidos de extrema derecha ofrecen una vía para reducir, o al menos para canalizar, las emociones negativas (Rydgren, 2003: 53).

Este es el caso del issue de la inmigración. Cuando los ciudadanos perciben la llegada de inmigrantes o sus efectos en la economía o en la seguridad ciudadana como un problema, pueden optar por votar a aquella organización política, que no sólo reintroduce en el debate político esta problemática, sino que da respuesta a los miedos y a las preocupaciones ciudadanas. 


\section{La extrema derecha y el issue de la inmigración}

Probablemente, más que con cualquier otro tema, la extrema derecha contemporánea se ha asociado con el issue de la inmigración (Hainsworth, 2008: 70). Algunos autores, incluso, han adoptado el término de "partidos antinmigrantes" o "partidos racistas" para identificar a un tipo específico de partidos de extrema derecha, sugiriendo que sus agendas se reducen únicamente a este tema y que su discurso apela al resentimiento popular en contra de los inmigrantes y de las políticas de inmigración de los gobiernos (Boomgaarden y Vliegenthart, 2007; Fennema, 1996: 26-27, 1997: 478-480; Van der Brug y Fennema, 2003, et al., 2000, 2005; van Spanje, 2017); y muchos otros autores han sustentado que los electores de estos partidos se diferencian de los de otras formaciones de su sistema político en el mantenimiento de actitudes negativas hacia la inmigración (Allen, 2017; Betz, 1994, 2001; Billiet y de Witte, 1995; Carter, 2005; Chapin, 1997; Cutts et al., 2011; Esses et al., 1998; Ivarsflaten, 2005, 2008; Kessler y Freeman, 2005; Lubbers, et al., 2002; Norris, 2009; Polyakova, 2015, Swyngedouw, 2001). Esto no significa que necesariamente los programas de los partidos de extrema derecha estén únicamente centrados en la inmigración (Betz, 2001), sino que sus votantes los identifican fundamentalmente con este tema específico.

Desde esta perspectiva, se postula que el éxito de los partidos de extrema derecha a finales de los años ochenta y noventa ha sido fundamentalmente el reflejo de la desafección pública con las políticas de inmigración, resultado del incremento sustancial del ratio de inmigrantes y del número de personas solicitantes de asilo y de refugiados en Europa occidental (Chapin, 1997), los cuales, a su vez, son una consecuencia, no sólo de la economía global y del desarrollo de las comunicaciones y de las redes de transporte (Esses et al. 1998), sino también de las políticas que habían paralizado la migración laboral.

El miedo, el resentimiento, la hostilidad o el odio hacia los inmigrantes se explica a través del aumento de la preocupación no sólo por el incremento del número de refugiados e inmigrantes ilegales, sino también por el cambio de la composición de las poblaciones de inmigrantes y refugiados. Como resultado, en muchos de los países de esta parte de Europa, la proporción de europeos occidentales ha permanecido estable, mientras que la población no europea se ha incrementado, lo que ha contribuido a la percepción de que Europa está siendo invadida por tradiciones, culturas y religiones extrañas, de modo que representan una amenaza a su propia cultura, costumbres y formas de vida (Betz, 1994; Lloyd, 2003). En Europa occidental, la demanda de restrictivas políticas de inmigración y de asilo derivan de la preocupación porque la diversidad religiosa, las tradiciones y el lenguaje tengan un impacto negativo sobre la unidad de la comunidad nacional (Ivarsflaten, 2005; Oesch, 2008). 
La gente que realmente se preocupa por la amenaza cultural que supone la inmigración, no cree que el voto a los partidos mainstreams sea un medio para modificar significativamente la política inmigratoria (Golder, 2003: 440).

Con la finalidad de presionar a los partidos del establishment para buscar una solución, cierto porcentaje de electores deliberadamente votan a los partidos de extrema derecha por sus severas políticas antinmigración. De este modo, el apoyo a la extrema derecha se puede interpretar como el uso racional y estratégico del voto para influir en las políticas públicas del gobierno con las que los votantes están en desacuerdo. Consecuentemente, la incapacidad de los partidos del establishment a la hora de proporcionar una respuesta al problema de la inmigración a lo largo del tiempo ha favorecido el desarrollo de los partidos de extrema derecha (Ignazi, 1992).

Además, este enfoque sugiere que los ciudadanos continúan apoyando a los partidos de extrema derecha precisamente porque el apoyo previo resultó ser efectivo. Una vez que la mayoría de los gobiernos imponen severas restricciones al flujo de inmigrantes (Akkerman, 2017; Helms, 1997; van Spanje, 2017), cuando están cerca de alcanzar el objetivo de inmigración cero, el sentimiento popular antinmigración no desaparece, sino que se desplaza a la cuestión de los beneficios sociales. Esto es especialmente llamativo en el caso de los solicitantes de asilo, quienes, con frecuencia, reciben apoyo financiero público (Arter, 1992). Si la xenofobia es el resultado del conflicto en torno a los recursos escasos entre los nativos y los inmigrantes, la proliferación de los impulsos racistas y del voto a la extrema derecha, puede interpretarse no sólo como una estrategia instrumental, sino como una reacción emotiva (Arzheimer, 2009: 260). En estas circunstancias, resulta sencillo para los partidos de extrema derecha apelar al miedo, a la ansiedad y a la aversión pública derivadas de la presunta carga que suponen los desempleados extranjeros y los refugiados en un ya estirado Estado de bienestar (Betz, 1990, 2002).

La relación entre la inmigración y la economía sugiere que, más que tratarse de programas neoliberales, los programas electorales de la extrema derecha están dominados por la economía nativista (Mudde, 2000, 2007). Es decir, la economía se interpreta como un servicio a la nación y sólo a la nación. Como consecuencia de la defensa, por parte de los partidos de extrema derecha, del principio de "preferencia nacional" con respecto al empleo y a la seguridad social (Oesch, 2008: 351), la economía nacional sería un servicio a los nativos (Eatwell, 2000), incluso debería estar bajo el estricto control de la nación. La economía nativista implica una visión nativista del Estado de bienestar, según la cual los servicios y beneficios sociales se encuentran garantizados para los nativos necesitados, siendo los extranjeros excluidos bajo el argumento de que el Estado de bienestar sólo puede sostenerse cuando se limita a los nativos. 
No obstante, el argumentario materialista matiza la relación entre la inmigración y la economía, al apuntar que no es necesario que los inmigrantes realmente causen desempleo, sino que la gente crea que sí lo hacen (Golder, 2003: 438). Se trata este de un tema que puede ser más una percepción que una realidad, es el miedo a los extranjeros más que su presencia lo que anima a los votantes a optar por la extrema derecha, por lo que los partidos de extrema derecha pueden, incluso, ser especialmente fuertes en áreas con bajos porcentajes de población inmigrante (Eatwell, 2017: 406). Los líderes de la extrema derecha, apelando al enfado y al resentimiento, tratan de convertir la satisfacción objetiva en la pobreza percibida (Mols y Jetten, 2016; Smith et al., 2012) a fin de captar el voto para sus organizaciones políticas.

En su discurso, los líderes de la extrema derecha también relacionan la presencia de extranjeros en las sociedades europeas occidentales con el aumento de la delincuencia y la criminalidad. Para la extrema derecha, el crimen es fundamentalmente un "alien" issue (un tema de extranjeros), en el sentido de que su discurso se centra en los crímenes cometidos por los no nativos (Mudde, 2019: 34). De acuerdo con estas formaciones, el crimen aumenta de manera incontrolada debido a las políticas cándidas e ineficaces de los políticos del establishment. Cuando su discurso es rebatido con datos que muestran que las ratios de crimen han realmente decrecido y que son relativamente reducidas, como es el caso de la mayoría de las sociedades de Europa occidental, la extrema derecha los desestima, calificándolos de mentiras pronunciadas por la élite corrupta para tapar los fallos la sociedad multicultural (Mudde, 2019). En este contexto, los partidos de extrema derecha defienden el endurecimiento de la ley y el orden, incluyendo el aumento de los dispositivos policiales, condenas más duras, la previsión de regímenes penitenciarios más severos y, en algunos casos, de la pena de muerte (Hainsworth, 2008), pero el freno real al aumento del crimen, de acuerdo con la extrema derecha, es el freno a la inmigración (Mudde, 2019).

\section{Análisis exploratorio de las percepciones de los votantes de VOX sobre el issue de la inmigración}

Gracias a la Encuesta Postelectoral de las Elecciones Generales de noviembre de 2019 realizada por el Equipo de Investigaciones Políticas (EIP) de la Universidade de Santiago de Compostela ${ }^{1}$, en la que se recogen una serie de cuestiones relativas al issue

1. La encuesta ha sido realizada entre el 14 de enero y el 22 de febrero de 2020 por el Equipo de Investigaciones Políticas de la USC, en colaboración con el laboratorio de encuestas telefónicas asistidas por ordenador de la Universidad de Granada, que llevó a cabo el trabajo de campo. El universo lo componen los ciudadanos españoles mayores de 18 años. El estudio posee un tamaño muestral de 1000 entrevistas, bajo el supuesto más desfavorable de $p=q$ y con un error asociado de $\pm 3,1 \%$ y con afijación proporcional (cuotas de sexo, de edad y provincia). 
de la inmigración (ver tabla Anexo: Variables incluidas en el análisis), es posible dar respuesta a la finalidad de esta investigación: observar las percepciones de los votantes de VOX en las Elecciones Generales de noviembre de 2019 acerca del issue de la inmigración.

En primer lugar, cuando se les pregunta a los votantes de VOX por el tema que les ha llevado a votar a esta formación política, casi el 38\% afirma que se trata de la propuesta consistente en un mayor control de la inmigración, lo que sitúa a este tema en la segunda posición, sólo por detrás de la Independencia de Cataluña. Esta posición resulta especialmente relevante si se tiene en cuenta que el porcentaje de población inmigrante censada se ha mantenido más o menos estable en los últimos diez años ${ }^{2}$.

\section{Gráfico 1}

Temas más importantes para la decisión del voto a VOX en las Elecciones Generales noviembre 2019 (Pregunta de respuesta múltiple)

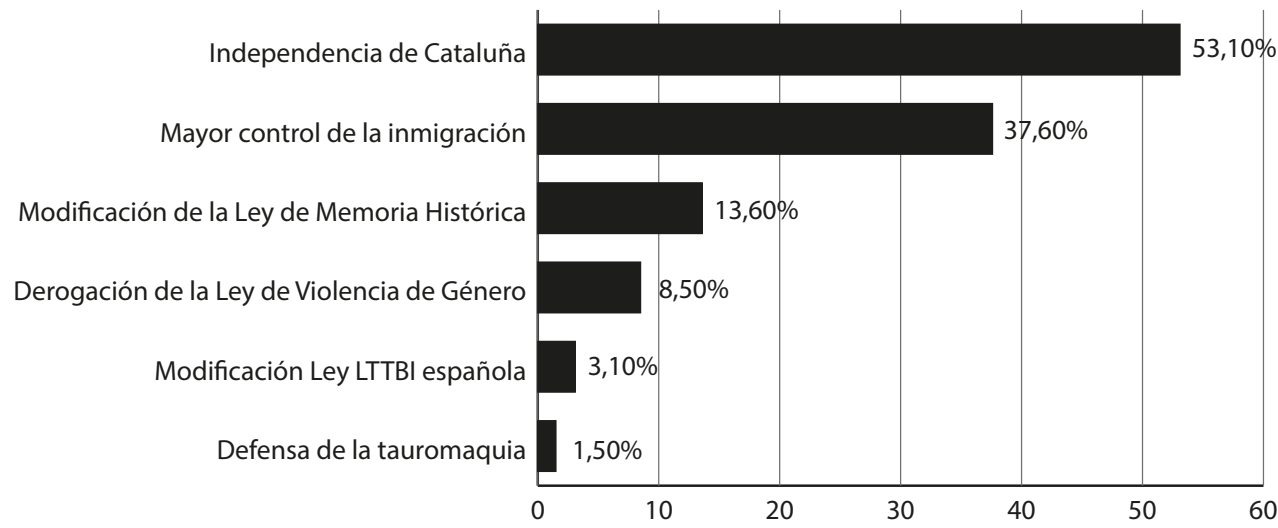

Fuente: elaboración propia a partir de los datos de la Encuesta Postelectoral Elecciones Generales noviembre 2019 realizada por el EIP

No obstante, el mayor control de la inmigración podría tratarse de una propuesta de aplicación equitativa al conjunto de la población extranjera o podría variar en función de la nacionalidad o de la raza de los inmigrantes. Cuando se pregunta por la medida en que España debería permitir que personas de la misma o diferente raza

2. De acuerdo con los datos del Instituto Nacional de Estadística (INE), en el año 2010 se alcanza el máximo histórico del porcentaje de población extranjera censada $(12,2 \%)$, cifra que ha ido paulatinamente descendiendo hasta el año 2018, cuando se produce un ligero repunte. 
o grupo étnico que la mayoría de los españoles, o que personas procedentes de países pobres no europeos vengan a vivir a España, existen diferencias en cuanto a la raza y procedencia de los inmigrantes y en función del voto de los ciudadanos en las pasadas Elecciones Generales. La tendencia pone de manifiesto que a medida que los electores votan a partidos que se ubican más a la derecha en la escala ideológica, son menos permisivos con la entrada de inmigrantes en España, y, mientras que en los votantes de los partidos de izquierdas (Unidas Podemos y PSOE), apenas existen diferencias en función de la raza o procedencia de los inmigrantes, en los partidos de derechas (C's y PP) el rechazo hacia los inmigrantes de distinta raza o procedentes de países pobres no europeos es ligeramente mayor que hacia los inmigrantes de la misma raza o grupo étnico que la mayoría de los españoles, acentuándose dichas diferencias en el caso de los votantes de VOX.

Tabla 1

Medida en la que España debería permitir que personas de la misma raza o grupo étnico que la mayoría de los españoles vengan a vivir a España por Recuerdo de Voto Elecciones Generales noviembre 2019

\begin{tabular}{|c|c|c|c|c|c|c|c|c|c|c|}
\hline & & & \multicolumn{7}{|c|}{ Recuerdo de Voto Elecciones Generales noviembre 2019} & \multirow[b]{2}{*}{ Total } \\
\hline & & & $\begin{array}{c}\text { Unidas } \\
\text { Podemos }\end{array}$ & PSOE & C's & PP & vox & Otros & $\mathrm{Ns} / \mathrm{Nc}$ & \\
\hline \multirow{5}{*}{ 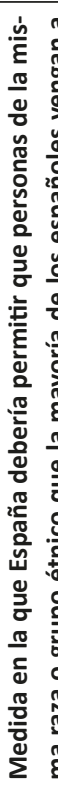 } & \multirow{5}{*}{ 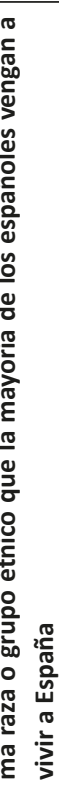 } & $\begin{array}{l}\text { Debería } \\
\text { permitírselo } \\
\text { a muchos }\end{array}$ & $76,9 \%$ & $64,8 \%$ & $37,3 \%$ & $32,4 \%$ & $23,8 \%$ & $60,7 \%$ & $51,1 \%$ & $54,5 \%$ \\
\hline & & $\begin{array}{l}\text { Debería } \\
\text { permitírselo } \\
\text { a algunos }\end{array}$ & $17,9 \%$ & $26,8 \%$ & $44,1 \%$ & $41,4 \%$ & $46,0 \%$ & $29,0 \%$ & $36,5 \%$ & $32,0 \%$ \\
\hline & & $\begin{array}{l}\text { Debería } \\
\text { permitírselo } \\
\text { a unos } \\
\text { pocos }\end{array}$ & $1,7 \%$ & $5,2 \%$ & $15,3 \%$ & $16,2 \%$ & $15,9 \%$ & $9,3 \%$ & $5,1 \%$ & $8,2 \%$ \\
\hline & & $\begin{array}{l}\text { No } \\
\text { permitírselo } \\
\text { a nadie }\end{array}$ & & $0,4 \%$ & $3,4 \%$ & $1,8 \%$ & $9,5 \%$ & & & $1,3 \%$ \\
\hline & & $\mathrm{Ns} / \mathrm{Nc}$ & $3,4 \%$ & $2,8 \%$ & & $8,1 \%$ & $4,8 \%$ & $0,9 \%$ & $7,3 \%$ & $4,0 \%$ \\
\hline \multicolumn{3}{|c|}{ Total } & $100,0 \%$ & $100,0 \%$ & $100,0 \%$ & $100,0 \%$ & $100,0 \%$ & $100,0 \%$ & $100,0 \%$ & $100,0 \%$ \\
\hline
\end{tabular}




\section{Tabla 2}

Medida en la que España debería permitir que personas de diferente raza o grupo étnico que la mayoría de los españoles vengan a vivir a España por Recuerdo de Voto Elecciones Generales noviembre 2019

\begin{tabular}{|c|c|c|c|c|c|c|c|c|c|}
\hline & & \multicolumn{7}{|c|}{ Recuerdo de Voto Elecciones Generales noviembre 2019} & \multirow[b]{2}{*}{ Total } \\
\hline & & $\begin{array}{l}\text { Unidas } \\
\text { Podemos }\end{array}$ & PSOE & C's & PP & vox & Otros & $\mathrm{Ns} / \mathrm{Nc}$ & \\
\hline \multirow{5}{*}{ 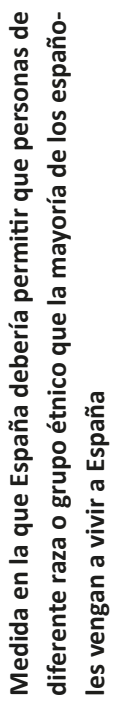 } & $\begin{array}{l}\text { Debería } \\
\text { permitírselo } \\
\text { a muchos }\end{array}$ & $75,4 \%$ & $64,8 \%$ & $35,0 \%$ & $28,8 \%$ & $22,2 \%$ & $61,7 \%$ & $44,1 \%$ & $52,5 \%$ \\
\hline & $\begin{array}{l}\text { Debería } \\
\text { permitírselo } \\
\text { a algunos }\end{array}$ & $17,8 \%$ & $26,8 \%$ & $43,3 \%$ & $41,4 \%$ & $46,0 \%$ & $29,0 \%$ & $40,4 \%$ & $32,5 \%$ \\
\hline & $\begin{array}{l}\text { Debería } \\
\text { permitírselo } \\
\text { a unos } \\
\text { pocos }\end{array}$ & $0,8 \%$ & $5,6 \%$ & $20,0 \%$ & $19,8 \%$ & $17,5 \%$ & $7,5 \%$ & $8,8 \%$ & $9,5 \%$ \\
\hline & $\begin{array}{l}\text { No } \\
\text { permitírselo } \\
\text { a nadie }\end{array}$ & $1,7 \%$ & $0,4 \%$ & $1,7 \%$ & $1,8 \%$ & $9,5 \%$ & $0,9 \%$ & $0,7 \%$ & $1,7 \%$ \\
\hline & $\mathrm{Ns} / \mathrm{Nc}$ & $4,2 \%$ & $2,4 \%$ & & $8,1 \%$ & $4,8 \%$ & $0,9 \%$ & $5,9 \%$ & $3,8 \%$ \\
\hline Total & & $100,0 \%$ & $100,0 \%$ & $100,0 \%$ & $100,0 \%$ & $100,0 \%$ & $100,0 \%$ & $100,0 \%$ & $100,0 \%$ \\
\hline
\end{tabular}

Fuente: elaboración propia a partir de los datos de la Encuesta Postelectoral Elecciones Generales noviembre 2019 realizada por el EIP 
Tabla 3

Medida en la que España debería permitir que personas procedentes de países pobres no europeos vengan a vivir a España por Recuerdo de Voto Elecciones Generales noviembre 2019

\begin{tabular}{|c|c|c|c|c|c|c|c|c|c|}
\hline & & \multicolumn{7}{|c|}{ Recuerdo de Voto Elecciones Generales noviembre 2019} & \multirow[b]{2}{*}{ Total } \\
\hline & & $\begin{array}{l}\text { Unidas } \\
\text { Podemos }\end{array}$ & PSOE & C's & PP & vox & Otros & $\mathrm{Ns} / \mathrm{Nc}$ & \\
\hline \multirow{5}{*}{ 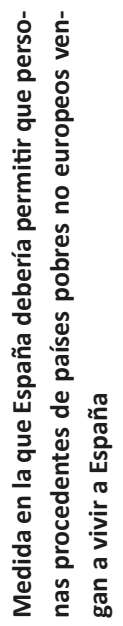 } & $\begin{array}{l}\text { Debería } \\
\text { permitírselo } \\
\text { a muchos }\end{array}$ & $76,3 \%$ & $64,8 \%$ & $35,0 \%$ & $27,9 \%$ & $19,0 \%$ & $61,7 \%$ & $41,9 \%$ & $52,0 \%$ \\
\hline & $\begin{array}{l}\text { Debería } \\
\text { permitírselo } \\
\text { a algunos }\end{array}$ & $16,9 \%$ & $26,4 \%$ & $43,3 \%$ & $42,3 \%$ & $49,2 \%$ & $28,0 \%$ & $39,0 \%$ & $32,3 \%$ \\
\hline & $\begin{array}{l}\text { Debería per- } \\
\text { mitírselo a } \\
\text { unos pocos }\end{array}$ & $2,5 \%$ & $6,0 \%$ & $20,0 \%$ & $17,1 \%$ & $15,9 \%$ & $8,4 \%$ & $9,6 \%$ & $9,6 \%$ \\
\hline & $\begin{array}{l}\text { No permitír- } \\
\text { selo a nadie }\end{array}$ & & $0,8 \%$ & $1,7 \%$ & $3,6 \%$ & $11,1 \%$ & $0,9 \%$ & $1,5 \%$ & $2,0 \%$ \\
\hline & $\mathrm{Ns} / \mathrm{Nc}$ & $4,2 \%$ & $2,0 \%$ & & $9,0 \%$ & $4,8 \%$ & $0,9 \%$ & $8,1 \%$ & $4,1 \%$ \\
\hline Total & & $100,0 \%$ & $100,0 \%$ & $100,0 \%$ & $100,0 \%$ & $100,0 \%$ & $100,0 \%$ & $100,0 \%$ & $100,0 \%$ \\
\hline
\end{tabular}

Fuente: elaboración propia a partir de los datos de la Encuesta Postelectoral Elecciones Generales noviembre 2019 realizada por el EIP

La misma tendencia se observa cuando los votantes declaran su grado de acuerdo con la gestión del Gobierno de las solicitudes de refugiados. De hecho, son los votantes de VOX los únicos que cuyo grado de acuerdo se encuentra por debajo de la puntuación media de cinco $(4,84)$ y más de dos puntos por debajo de la media del conjunto de los votantes $(7,16)$. 
Tabla 4

Grado de acuerdo con la gestión del Gobierno de las solicitudes de refugiados por Recuerdo de Voto Elecciones Generales noviembre 2019

\begin{tabular}{lccc}
\hline $\begin{array}{c}\text { Recuerdo de Voto Elecciones Generales } \\
\text { noviembre 2019 }\end{array}$ & Media & N & $\begin{array}{c}\text { Desviación } \\
\text { estándar }\end{array}$ \\
\hline Unidas Podemos & 8,52 & 114 & 2,153 \\
\hline PSOE & 7,89 & 238 & 2,170 \\
\hline C's & 6,59 & 59 & 2,607 \\
\hline PP & 5,75 & 105 & 2,571 \\
\hline VOX & 4,84 & 60 & 3,036 \\
\hline Otros & 7,29 & 108 & 2,374 \\
\hline Ns/Nc & 7,00 & 127 & 2,245 \\
\hline Total & 7,16 & 811 & 2,582 \\
\hline \hline
\end{tabular}

Fuente: elaboración propia a partir de los datos de la Encuesta Postelectoral Elecciones Generales noviembre 2019 realizada por el EIP

Esto nos lleva a plantearnos si los votantes de VOX se sienten exclusivamente preocupados por la inmigración o si también tienen la percepción de que la presencia de inmigrantes tiene consecuencias devastadoras para la economía o para la seguridad ciudadana. En lo que respecta a la economía, si bien los votantes de VOX son los que peor valoran el efecto de los inmigrantes en la economía española y son los que consideran, en mayor medida, que los inmigrantes "roban" el trabajo a los españoles, en el aspecto en el que sus electores muestran una posición más extrema es en la cuestión más relacionada con los servicios sociales: los votantes de VOX consideran que los inmigrantes reciben servicios sanitarios y del Estado de Bienestar en mayor medida de lo que aportan mediante su trabajo y el pago de impuestos, posicionándose casi un punto por encima de los votantes del siguiente partido situado más a la derecha del espectro ideológico, el PP. 
Tabla 5

Economía nativista por Recuerdo de Voto Elecciones Generales noviembre 2019

\begin{tabular}{|c|c|c|c|c|}
\hline \multicolumn{2}{|c|}{$\begin{array}{l}\text { Recuerdo de Voto Elecciones } \\
\text { Generales noviembre } 2019\end{array}$} & $\begin{array}{l}\text { Valoración del efecto } \\
\text { en la economía espa- } \\
\text { ñola de que la gente } \\
\text { de otros países venga } \\
\text { a vivir a España }\end{array}$ & $\begin{array}{l}\text { Medida en la que la } \\
\text { gente que viene a } \\
\text { vivir a España "roba" } \\
\text { el trabajo a los traba- } \\
\text { jadores españoles o } \\
\text { ayuda a crear nuevos } \\
\text { puestos de trabajo }\end{array}$ & $\begin{array}{l}\text { Medida en la que la } \\
\text { gente que viene a } \\
\text { vivir a España recibe } \\
\text { más de lo que aporta } \\
\text { o aporta más de lo } \\
\text { que recibe }\end{array}$ \\
\hline \multirow{3}{*}{$\begin{array}{l}\text { Unidas } \\
\text { Podemos }\end{array}$} & Media & 1,86 & 2,22 & 3,23 \\
\hline & $\mathrm{N}$ & 117 & 111 & 114 \\
\hline & Desviación estándar & 2,091 & 2,470 & 2,532 \\
\hline \multirow{3}{*}{ PSOE } & Media & 2,31 & 2,80 & 3,99 \\
\hline & $\mathrm{N}$ & 242 & 236 & 234 \\
\hline & Desviación estándar & 2,036 & 2,578 & 2,645 \\
\hline \multirow{3}{*}{$C^{\prime} s$} & Media & 3,24 & 3,71 & 5,27 \\
\hline & N & 60 & 57 & 59 \\
\hline & Desviación estándar & 2,014 & 2,592 & 2,822 \\
\hline \multirow{3}{*}{ PP } & Media & 3,77 & 4,50 & 6,08 \\
\hline & $\mathrm{N}$ & 106 & 107 & 108 \\
\hline & Desviación estándar & 2,248 & 2,462 & 2,550 \\
\hline \multirow{3}{*}{ VOX } & Media & 4,63 & 4,94 & 7,06 \\
\hline & $\mathrm{N}$ & 60 & 59 & 61 \\
\hline & Desviación estándar & 2,876 & 2,680 & 2,499 \\
\hline \multirow{3}{*}{ Otros } & Media & 3,03 & 3,14 & 4,14 \\
\hline & $N$ & 107 & 105 & 103 \\
\hline & Desviación estándar & 2,387 & 2,155 & 2,309 \\
\hline \multirow{3}{*}{$\mathrm{Ns} / \mathrm{Nc}$} & Media & 3,08 & 3,49 & 4,74 \\
\hline & $\mathrm{N}$ & 132 & 123 & 122 \\
\hline & Desviación estándar & 2,254 & 2,288 & 2,346 \\
\hline \multirow{3}{*}{ Total } & Media & 2,89 & 3,32 & 4,62 \\
\hline & $\mathrm{N}$ & 824 & 797 & 800 \\
\hline & Desviación estándar & 2,343 & 2,581 & 2,751 \\
\hline
\end{tabular}

Fuente: elaboración propia a partir de los datos de la Encuesta Postelectoral Elecciones Generales noviembre 2019 realizada por el EIP. 
Tabla 6

Medida en la que considera que los inmigrantes empeoran o mejoran los niveles de delincuencia en España por Recuerdo de Voto Elecciones Generales noviembre 2019

\begin{tabular}{lccc}
\hline $\begin{array}{c}\text { Recuerdo de Voto Elecciones } \\
\text { Generales noviembre 2019 }\end{array}$ & Media & N & $\begin{array}{c}\text { Desviación } \\
\text { estándar }\end{array}$ \\
\hline Unidas Podemos & 2,93 & 114 & 2,728 \\
\hline PSOE & 3,68 & 236 & 2,808 \\
\hline C's & 4,72 & 58 & 2,830 \\
\hline PP & 5,78 & 106 & 2,660 \\
\hline VOX & 7,08 & 59 & 2,870 \\
\hline Otros & 4,22 & 102 & 2,695 \\
\hline Ns/Nc & 4,48 & 126 & 2,693 \\
\hline Total & 4,37 & 801 & 2,961 \\
\hline \hline
\end{tabular}

Fuente: elaboración propia a partir de los datos de la Encuesta Postelectoral Elecciones Generales noviembre 2019

Sin embargo, la diferencia entre los votantes de VOX y los votantes del PP es todavía mayor cuando se relaciona la inmigración con la seguridad ciudadana. En una escala de cero a diez, los votantes de VOX se sitúan por encima del siete cuando se les pregunta su grado de acuerdo con la afirmación de que los inmigrantes empeoran los niveles de delincuencia en España.

\section{Conclusiones}

La inmigración no sólo es un issue clave para los partidos de extrema derecha, sino que es un tema que también resulta crucial para los votantes de estos partidos políticos. Y los votantes de VOX no parecen ser la excepción.

Más allá de la hostilidad de los votantes de VOX hacia los inmigrantes, sobre todo, los de distinta raza o los procedentes de países pobres no europeos, resulta especialmente relevante la preocupación por la gestión del Gobierno de las solicitudes de asilo, probablemente debido a la decisión del Gobierno de acoger a los refugiados rescatados por el Open Arms.

En consonancia con esta preocupación, los votantes de VOX no sólo consideran que los inmigrantes "roben" los puestos de trabajo a los españoles, produciendo efectos devastadores en la economía, sino que, sobre todo, los inmigrantes son percibidos como una carga económica para los servicios sociales y una amenaza para la seguridad ciudadana. 
Los votantes de esta formación política, al igual que los de sus homólogos europeos, parecen haber recibido a las nuevas remesas de inmigrantes con una mezcla de aprensión, desconfianza y desdén. Estos sentimientos se traducen en una ola de ansiedad y resentimiento, emociones que, sin atarlas a términos directamente racistas, VOX, siguiendo el estilo de otras organizaciones de extrema derecha, ha transformado en beneficios electorales.

En su lugar, se han hecho eco de las críticas a la política de refugiados del Gobierno de Pedro Sánchez, centrando la atención pública en la creciente carga financiera que los refugiados suponen para España. Esto impacta doblemente en la conciencia de la sociedad española: los inmigrantes no sólo son una carga económica para los servicios sociales, sino que también arrebatan los escasos puestos de trabajo a los españoles desempleados. Además, los inmigrantes son los responsables, a ojos de los votantes de VOX, del incremento de las ratios de delincuencia.

Santiago Abascal y el resto de los dirigentes de VOX se esfuerzan por relacionar en su discurso la inmigración con la delincuencia, culpando a los extranjeros del incremento de las agresiones a las mujeres o poniendo el foco en las revueltas de los MENAs (Menores Extranjeros No Acompañados). Discurso que, a tenor de los datos, parecer tener consecuencias en las percepciones de sus votantes.

En otras palabras, a expensas de la realización de un análisis inferencial, parece que el discurso de VOX ha sido exitoso a la hora de convertir la satisfacción objetiva en la pobreza y en la delincuencia percibida, logrando su fin último: la captación del voto en las Elecciones Generales de noviembre de 2019.

\section{Bibliografía}

AKKERMAN, Tjitske (2017) "Comparing Radical Right Parties in Government: Immigration and Integration Policies in Nine Countries (1996-2010)", MUDDE, Cas (ed.) The Populist Radical Right. A Reader. New York: Routledge, 493-507

ALLEN, Trevor J. (2017) "All in the Party Family? Comparing Far Right Voters in Western and Post-Communist Europe", Party Politics, 23(3), 274-285. En: https:// doi.org/10.1177/1354068815593457

ARTER, David (1992) "Black Faces in the Blond Crowd: Populism Racialism in Scandinavia”, Parliamentary Affairs, 45(3), 357-372. En: https:/doi.org/10.1093/ oxfordjournals.pa.a052364

ARZHEIMER, Kay (2009) "Contextual Factors and the Extreme Right Vote in Western Europe, 1980-2002”, American Journal of Political Science, 53(2), 259275. En: https://doi.org/10.1111/j.1540-5907.2009.00369.x

BETZ, Hans-George (1990) "Politics of Resentment. Right-wing Radicalism in West Germany”, Comparative Politics, 23, 45-60. En: https://doi.org/10.2307/422304 
BETZ, Hans-George (1993) “The New Politics of Resentment: Radical Right-Wing Populist Parties in Western Europe" Comparative Politics, 25(4), 413-427. En: https://doi.org/10.2307/422034

BETZ, Hans-George (1994) Radical Right-Wing Populism in Western Europe. Londres: McMillan

BETZ, Hans-George (2001) "Exclusionary Populism in Austria, Italy and Switzerland”, International Journal, 56(3), 393-420. En: https:/doi. org/10.1177/002070200105600302

BETZ, Hans-George (2002) "Conditions Favoring the Success (and Failure) of Radical Right-Wing Populist Parties in Contemporary Democracies”, MENY, Yves e Yves, SUREL (ed.) Democracies and the Populist Challenge. Basingstoke: Palgrave, 197-213

BILLIET, Jaak y Hans DE WITTE (1995) "Attitudinal Dispositions to Vote for a "New" Extreme Right Party: The Case of the "Vlaams Block"', European Journal of Political Research, 27(2), 181-202. En: https://doi.org/10.1111/j.1475-6765.1995. tb00635.x

BOOMGAARDEN, Hajo G. y Rens VLIEGENTHART (2007) "Explaining the Rise of Anti-Immigrant Parties: The Role of News Media Content in the Netherlands", Electoral Studies, 26(2), 404-417. En: https://doi.org/10.1016/j. electstud.2006.10.018

CARTER, Elisabeth (2005) The Extreme Right in Western Europe. Success or Failure? Manchester: Manchester University Press

CHAPIN, Wesley D. (1997) "Explaining the Electoral Success of the New Right: The German Case", West European Politics, 20(2), 53-72. En: https://doi. org/10.1080/01402389708425191

CUTTS, David, Robert FORD y Matthew J. GOODWIN (2011) "Anti-Immigrant, Politically Disaffected or Still Racist After All? Examining the Attitudinal Drivers of Extreme Right Support in Britain in the 2009 European Elections", European Journal of Political Research, 50, 418-440. En: https://doi.org/10.1111/j.14756765.2010.01936.x

EATWELL, Roger (2000) “The Rebirth of the "Extreme Right" in Western Europe", Parliamentary Affairs, 53(3), 407-425. En: https://doi.org/10.1093/pa/53.3.407

EATWELL, Roger (2017) “Ten Theories of the Extreme Right”, MUDDE, Cas (ed.) The Populist Radical Right. A Reader. New York: Routledge, 404-423

ESSES, Victoria M., Lynne M. JACKSON y Tamara L. AMSTRONG (1998) "Intergroup Competition and Attitudes Toward Immigrants and Immigration: An Instrumental Model of Group Conflict”, Journal of Social Issues, 54(4), 699724. En: https://doi.org/10.1111/j.1540-4560.1998.tb01244.x 
FENNEMA, Meindert (1996) Some Theorical Problems and Issues in Comparison of Anti-Immigrant Parties in Western Europe. Barcelona: Institut de Ciències Polítiques I Socials

FENNEMA, Meindert (1997) "Some Conceptual Issues and Problems in the Comparison of Anti-Immigration Parties in Western Europe", Party Politics, 3, 473-492. En: https://doi.org/10.1177/1354068897003004002

GOLDER, Matt (2003) "Explaining Variation in the Success of Extreme Right Parties in Western Europe", Comparative Political Studies, 36(4), 432-466. En: https:// doi.org/10.1177/0010414003251176

HAINSWORTH, Paul (2008) The Extreme Right in Western Europe. New York: Routledge.

HELMS, Ludger (1997) "Right-Wing Populist Parties in Austria and Switzerland: A Comparative Analysis of Electoral Support and Conditions of Success", West European Politics, 20(2), 37-52. En: https://doi.org/10.1080/01402389708425190

IGNAZI, Piero (1992) “The Silent Counter-Revolution. Hypotheses on the Emergence of Extreme Right-Wing Parties in Europe", European Journal of Political Research, 22, 3-34. En: https://doi.org/10.1111/j.1475-6765.1992.tb00303.x

IVARSFLATEN, Elisabeth (2005) "Threatened by Diversity: Why Restrictive Asylum and Immigration Policies Appeal to Voters in Western Europe", Journal of Elections, Public Opinion and Parties, 15(1), 21-45. En: https://doi. org/10.1080/13689880500064577

IVARSFLATEN, Elisabeth (2008) "What Unites the Populist Right in Western Europe? Reexamining Grievance Mobilization Models in Seven Successful Cases", Comparative Political Studies, 41(1), 3-23. En: https://doi. org/10.1177/0010414006294168

KESSLER, Alan E. y Gary P. FREEMAN (2005) "Support for the Extreme RightWing Parties in Western Europe: Individual Attributes, Political Attitudes, and National Context", Comparative European Politics, 3, 261-288. En: https://doi. org/10.1057/palgrave.cep.6110063

KITSCHELT, Herbert (1995) The Radical Right in Western Europe: A Comparative Analysis. Ann Arbor: The University of Michigan Press

LLOYD, John (2003) “The Closing of the European Gates? The New Populist Parties of Europe", Political Quarterly, 74(10), 88-99. En: https://doi.org/10.1111/j.1467923X.2003.00583.x

LUBBERS, Marcel, Mérove GIJSBERTS y Peer SCHEEPERS (2002) "Extreme RightWing Voting in Western Europe", European Journal of Political Research, 41, 345378. En: https://doi.org/10.1111/1475-6765.00015 
MEGUID, Bonnie M. (2005) "Competition Between Unequals: The Role of Mainstream Party Strategy in Niche Party Success", American Political Science Review, 99(3), 347-359. En: https://doi.org/10.1017/S0003055405051701

MOLS, Frank y Jolanda JETTEN (2016) "Explaining the Appeal of Populist Right Wing Parties in Times of Economic Prosperity”, Political Psychology, 37(2), 275292. En: https://doi.org/10.1111/pops.12258

MUDDE, Cas (2000) The Ideology of the Extreme Right. Manchester: Manchester University Press

MUDDE, Cas (2007) Populist Radical Right Parties in Europe. Cambridge: Cambridge University Press

MUDDE, Cas (2017) “The 2012 Stein Rokkan Lecture: Three Decades of Populist Radical Right Parties in Western Europe: So What?" MUDDE, Cas (ed.) The Populist Radical Right. A Reader. New York: Routledge, 527-540

MUDDE, Cas (2019) The Far Right Today. John Wiley \& Sons

NORRIS, Pippa (2009) Derecha radical: votantes y partidos políticos en el mercado electoral. Madrid: Akal

OESCH, Daniel (2008) “Explaining Workers'Support for Right-Wing Populist Parties in Western Europe: Evidence from Austria, Belgium, France, Norway, and Switzerland", International Political Science Review, 29(3), 349-373. En: https://doi.org/10.1177/0192512107088390

POLYAKOVA, Alina (2015) “The Backward East? Explaining Differences in Support for Radical Right Parties in Western and Eastern Europe", Journal of Comparative Politics, 8(1), 49-74

RYDGREN, Jens (2003) "Meso-Level Reasons for Racism and Xenophobia. Some Converging and Diverging Effects of Radical Right Populism in France and Sweden", European Journal of Social Theory, 6, 45-68. En: https://doi. org/10.1177/1368431003006001560

SMITH, Heather J., Thomas F. PETTIGREW, Gina M. PIPPIN y Silvana BIALOSIEWICZ (2012) "Relative Deprivation: A Theorical and Meta-Analytic Review” Personality and Social Psychology Review, 16(3), 203-232. En: https:// doi.org/10.1177/1088868311430825

SWYNGEDOUW, Marc (2001) “The Subjective, Cognitive and Affective Map of Extreme Right Votes: Using Open-Ended Questions in Exist Polls” Electoral Studies, 20(2), 217-241. En: https://doi.org/10.1016/S0261-3794(00)00010-X

VAN DER BRUG, Wouter, Meindert FENNEMA y Jean TILLIE (2000) "Antiimmigrant Parties in Europe: Ideological or Protest Vote?", European Journal of Political Research, 37(2), 77-102. En: https://doi.org/10.1111/1475-6765.00505 
VAN DER BRUG, Wouter y Meindert FENNEMA (2003) "Protest or Mainstream? How the European Anti-Inmigrant Parties Developed into Two Separate Groups by 1999", European Journal of Political Research, 42(1), 55-76. En: https://doi. org/10.1111/1475-6765.00074

VAN DER BRUG, Wouter, Meindert FENNEMA y Jean TILLIE (2005) "Why Some Anti-Immigrant Parties Fail and Others Succeed: A Two-Step Model of Aggregate Electoral Support", Comparative Political Studies, 38(5), 537-573. En: https://doi.org/10.1177/0010414004273928

VAN SPANJE, Joost (2017) "Contagious Parties: Anti-Immigration Parties and their Impact on the Other Parties" MUDDE, Cas (ed.) The Populist Radical Right. A Reader. New York: Routledge, 474-492

ZÚQUETE, José Pedro (2017) "The European Extreme-Right and Islam: New Directions?" MUDDE, Cas (ed.) The Populist Radical Right. A Reader. New York: Routledge, 103-123 


\section{Anexo: Variables incluidas en el análisis}

\begin{tabular}{|c|c|c|}
\hline Variable & Tipo & Interpretación \\
\hline $\begin{array}{l}\text { Recuerdo de Voto } \\
\text { Elecciones Generales } \\
\text { noviembre } 2019\end{array}$ & $\begin{array}{l}\text { Categórica con } 7 \\
\text { categorías }\end{array}$ & $\begin{array}{l}\text { 1: Voto Unidas Podemos } \\
\text { 2: Voto PSOE } \\
\text { 3: Voto C's } \\
\text { 4: Voto PP } \\
\text { 5: Voto VOX } \\
\text { 6: Voto Otros } \\
\text { 7: Ns/Nc }\end{array}$ \\
\hline $\begin{array}{l}\text { Temas más importantes } \\
\text { para la decisión del voto } \\
\text { a VOX }\end{array}$ & $\begin{array}{l}\text { Categórica con } \\
9 \text { categorías } \\
\text { (Pregunta de res- } \\
\text { puesta múltiple) }\end{array}$ & $\begin{array}{l}\text { 1: Independencia de Cataluña } \\
\text { 2: Mayor control de la inmigración } \\
\text { 3: Modificación de la ley de Memoria Histórica } \\
\text { 4: Derogación de la Ley de Violencia de Género } \\
\text { 5: Modificación Ley LGTBI española } \\
\text { 6: Defensa de la tauromaquia } \\
\text { 7: Otro } \\
\text { 8: Ninguno } \\
\text { 9: Ns/Nc }\end{array}$ \\
\hline $\begin{array}{l}\text { Posicionamiento antes } \\
\text { multiculturalismo / } \\
\text { inmigración }\end{array}$ & Continua & $\begin{array}{l}\text { 0: Considera muy positivo que en un país convivan perso- } \\
\text { nas de diferente origen, cultura y religión } \\
\text { 10: Considera que la presencia de inmigrantes puede poner } \\
\text { en peligro los valores y la cultura del país }\end{array}$ \\
\hline $\begin{array}{l}\text { Medida en la que Espa- } \\
\text { ña debería permitir que } \\
\text { personas de la misma } \\
\text { raza o grupo étnico } \\
\text { que la mayoría de los } \\
\text { españoles vengan a vivir } \\
\text { a España }\end{array}$ & Ordinal & $\begin{array}{l}\text { 1: Debería permitírselo a muchos } \\
\text { 2: Debería permitírselo a algunos } \\
\text { 3: Debería permitírselo a unos pocos } \\
\text { 4: No permitírselo a nadie } \\
\text { 5: Ns/Nc }\end{array}$ \\
\hline $\begin{array}{l}\text { Medida en la que Espa- } \\
\text { ña debería permitir que } \\
\text { personas de diferente } \\
\text { raza o grupo étnico } \\
\text { que la mayoría de los } \\
\text { españoles vengan a vivir } \\
\text { a España }\end{array}$ & Ordinal & $\begin{array}{l}\text { 1: Debería permitírselo a muchos } \\
\text { 2: Debería permitírselo a algunos } \\
\text { 3: Debería permitírselo a unos pocos }\end{array}$ \\
\hline
\end{tabular}




\begin{tabular}{|c|c|c|}
\hline Variable & Tipo & Interpretación \\
\hline $\begin{array}{l}\text { Medida en la que Espa- } \\
\text { ña debería permitir que } \\
\text { personas procedentes } \\
\text { de países pobres no eu- } \\
\text { ropeos vengan a vivir a } \\
\text { España }\end{array}$ & Ordinal & $\begin{array}{l}\text { 1: Debería permitírselo a muchos } \\
\text { 2: Debería permitírselo a algunos } \\
\text { 3: Debería permitírselo a unos pocos } \\
\text { 4: No permitírselo a nadie } \\
\text { 5: Ns/Nc }\end{array}$ \\
\hline $\begin{array}{l}\text { Grado de acuerdo con la } \\
\text { gestión del Gobierno de } \\
\text { las solicitudes de refu- } \\
\text { giados }\end{array}$ & Continua & $\begin{array}{l}\text { 0: Totalmente en desacuerdo } \\
\text { 10: Totalmente de acuerdo }\end{array}$ \\
\hline $\begin{array}{l}\text { Valoración del efecto en } \\
\text { la economía española } \\
\text { de que la gente de otros } \\
\text { países venga a vivir a Es- } \\
\text { paña }\end{array}$ & Continua & $\begin{array}{l}\text { 0: Bueno para la economía española que la gente de otros } \\
\text { países venga a vivir a España } \\
\text { 10: Malo para la economía española que la gente de otros } \\
\text { países venga a vivir a España }\end{array}$ \\
\hline $\begin{array}{l}\text { Medida en la que la gen- } \\
\text { te que viene a vivir a Es- } \\
\text { paña "roba" el trabajo a } \\
\text { los trabajadores españo- } \\
\text { les o ayuda a crear nue- } \\
\text { vos puestos de trabajo }\end{array}$ & Continua & $\begin{array}{l}\text { 0: La gente que viene a vivir a España ayudan a crear nuevos } \\
\text { puestos de trabajo } \\
\text { 10: La gente que viene a vivir a España "roba" el trabajo a los } \\
\text { trabajadores españoles }\end{array}$ \\
\hline $\begin{array}{l}\text { Medida en la que la gen- } \\
\text { te que viene a vivir a } \\
\text { España recibe más de lo } \\
\text { que aporta o aporta más } \\
\text { de lo que recibe }\end{array}$ & Continua & $\begin{array}{l}\text { 0: Aporta más de lo que recibe } \\
\text { 10: Recibe más de lo que aporta }\end{array}$ \\
\hline $\begin{array}{l}\text { Medida en la que consi- } \\
\text { dera que los inmigrantes } \\
\text { empeoran o mejoran los } \\
\text { niveles de delincuencia } \\
\text { en España }\end{array}$ & Continua & $\begin{array}{l}\text { 0: Totalmente en desacuerdo } \\
\text { 10: Totalmente de acuerdo }\end{array}$ \\
\hline
\end{tabular}

Fuente: elaboración propia. 